\title{
The synergistic effects of green tea polyphenols and antibiotics against potential pathogens
}

\author{
Bobak Haghjoo $^{1}$, Lee H. Lee ${ }^{1}$, Umme Habiba ${ }^{1}$, Hassan Tahir ${ }^{1}$, Moe Olabi ${ }^{1}$, Tin-Chun Chu ${ }^{2}$ \\ ${ }^{1}$ Department of Biology \& Molecular Biology, Montclair State University, Montclair, USA \\ ${ }^{2}$ Department of Biological Sciences, Seton Hall University, South Orange, USA \\ Email: leel@mail.montclair.edu
}

Received 28 July 2013; revised 28 August 2013; accepted 15 September 2013

Copyright (C) 2013 Bobak Haghjoo et al. This is an open access article distributed under the Creative Commons Attribution License, which permits unrestricted use, distribution, and reproduction in any medium, provided the original work is properly cited.

\begin{abstract}
Green tea leaves contain many polyphenolic compounds such as (-)-epicatechin (EC), (-)-epicatechin-3gallate (ECG), (-)-epigallocatechin (EGC), and (-)epigallocatechin-3-gallate (EGCG). These polyphenol compounds have been implicated to have distinct properties that combat the harmful effects of cell proliferation. They contain certain anti-viral and antibacterial properties that inhibit growth. In this study, $1 \%$ green tea and modified lipophilic green tea polyphenols (GTP and LTP) were used in combination with the most commonly prescribed antibiotics to study their effects on gram-positive, gram-negative, and acid-fast bacteria. The results indicated that $1 \%$ GTP and 1\% LTP provided different synergistic effects on several antibiotics in various bacteria. It was found that 1\% GTP works the best synergistically against Enterobacter aerogenes, making the resistant strain susceptible to 8 out of 12 antibiotics used. 1\% LTP worked the best on Escherichia coli and was able to convert 7 antibiotic resistant categories to susceptible. In addition, $1 \%$ LTP was also able to inhibit the growth of Serratia marcescens synergistically with 3 antibiotics. These results suggest that $1 \%$ GTP and $1 \%$ LTP provide beneficial effects on selected antibiotics against microbial growth and are able to reverse the antibiotic resistance to susceptible. Green tea polyphenols could serve as natural alternatives to combat against antibiotic resistance pathogens.
\end{abstract}

Keywords: GTP; LTP; Green Tea Polyphenols; Pathogenic Microorganisms; Kirby-Bauer Disk Diffusion Method; Antibiotic Resistance

\section{INTRODUCTION}

Chinese green tea is an orally ingested beverage popular in Asian and Western communities. The tea is derived from an herbal plant Camellia sinensis [1]. The greatest cultivation of this plant is mainly in Mainland China and the areas that surrounding it. South and Southeast Asia recorded green tea as the second most popular beverage consumed worldwide after water [2]. Green tea has been observed to have several medical benefits, some of which include reduction in cholesterol level, protection against cardio-vascular diseases, cancer, etc. [3]. Polyphenolic compounds found exclusively in green tea have been implicated to have distinct properties that combat the harmful effects of many potentially pathogenic bacteria $[4,5]$.

The polyphenolic compound that has been attributed to the positive claims of green tea is known as Epigallocatechin-3-gallate (EGCG). This pure compound is relatively expensive and unstable. It can be oxidized rapidly in normal atmospheric conditions and is not lipid-soluble. Since, EGCG has poor membrane permeability, low chemical stability and is usually metabolized rapidly [6], it loses its abilities long before one would be able to apply it. Most of the studies with EGCG have to be conducted with freshly prepared EGCG otherwise it loses its potent antimicrobial activity [7]. This poses an issue since EGCG has been reported to have many medical benefits. The green tea polyphenols need to be modified to form a lipophilic tea polyphenol (LTP), which is soluble in any lipid medium [8]. This would allow for increased utilization to be employed as a topical application in solution.

Chinese green tea extract has also been found to strongly inhibit the growth of major food-borne pathogens, Escherichia coli O157:H7, Salmonella typhimurium DT104, Listeria monocytogenes, Staphylococcus aureus, and a diarrhea food poisoning bacteria Bacillus cereus in varied levels of effectiveness [9]. This is of particular interest since EGCG has been suggested to be highly effective against $S$. aureus and also methicillin 
resistant S. aureus (MRSA). S. aureus is of major concern to the dairy industry worldwide since it has been associated with bovine mastitis in high rates causing tremendous economic losses. This pathogen has become an increasingly problematic infection with the response in evolutionary resistance to a broad spectrum of antibiotics. As a positive marker of a recent study, it was concluded that after about 5 to 6 hours of incubation under assay conditions, $500 \mu \mathrm{g} / \mathrm{ml}$ of green tea extract was able to completely inhibit the growth of both susceptible and resistant strains of this bacteria [9].

In this study, green tea polyphenols (GTP) and lipophilic tea polyphenols (LTP) were used in combination with twelve of the antibiotic disks to evaluate and establish a profile for their antimicrobial activities in different groups of microorganisms. GTP is a crude extract of the polyphenols found in green tea and is a cheaper alternative to using EGCG. It has a marked $70 \%$ or higher purity. LTP is an esterified version of EGCG and is lipidsoluble. This makes it an ideal candidate to be utilized in topical solutions or ointments to enhance treatment. The evaluation gives insight into understanding the effects of novel tea compounds and its efficacy in conjunction with antibiotics in vitro. This may be used as an alternative therapeutic agent or synergistic agent to battle antibiotic resistant bacterial infections in the future.

\section{METHODS}

\subsection{Culture Strains}

There were six microorganisms in stock, constantly maintained and utilized in the experiment. The organisms were gram positive bacteria: Staphylococcus epidermidis, Bacillus megaterium; gram negative bacteria: Escherichia coli, Serratia marcescens, Enterobacter aerogenes; and an acid-fast bacterium Mycobacterium smegmatis. They were used to screen and establish profile in this study. All the cultures were maintained in nutrient broth, nutrient agar plate, Muller-Hinton agar or Muller-Hinton broth. They were grown at $37^{\circ} \mathrm{C}$ incubation with consistent shaking at $250 \mathrm{rpm}$ except Serratia marcescens which is kept at room temp. The overnight cultures were used in this study. Fresh stocks were prepared and stored at $4^{\circ} \mathrm{C}$ and permanent stocks were kept in $-80^{\circ} \mathrm{C}$. The abbreviations for the microorganisms are, in the same order as mentioned above, as noted to be the following: S. epidermidis, B. megaterium, E. coli, S. marcescens, E. aerogenes, and M. smegmatis.

\subsection{Preparation of GTP and LTP}

The preparation of GTP and modified LTP was utilized by creating stock solution from fine tea powder. The GTP was dissolved in water at the final concentration of $1 \%$ and the LTP was dissolved in $100 \%$ ethanol at the final concentration of $1 \%$. The solution then went through a $0.45 \mu \mathrm{m}$ filter unit to obtain a sterile solution.

\subsection{Disk Diffusion Method}

The experiment utilized the Kirby-Bauer disk diffusion method. After inoculation and stamping of the antibiotics, $25 \mu 1$ of $1 \%$ GTP or LTP was directly infused with the filter disk at room temperature. The antibiotics alone were used as control for each microorganism. The plates were incubated at 24 and 48 hours at $37^{\circ} \mathrm{C}$. The zones of inhibition were measured in millimeters across their diameter of their clear region. This region included the antibiotic disk itself and utilized minimum-detection limit (MDL) as shown in Figure 1. The zones of inhibition (ZOI) were categorized into three unique categories that effectively informed the status of the effectiveness against the microorganism. The three categories are susceptible (S), intermediate (I), and resistant (R) which are defined in Table 1 with its respective abbreviation [10, 11]. The antibiotic disks included, in alphabetical order, are: Ampicillin (AM10), Bacitracin (B10), Cephalothin (CF30), Chloramphenicol (C30), Doxycycline (D10), Erythromycin (E15), Gentamicin (GM10), Penicillin (P10), Polymyxin (PB300), Rifampin (RA5), Streptomycin (S10), and Tetracycline (TE30). Three repeating with or without tea polyphenols (GTP or LTP) of each experiment were carried out and the results were shown by the mean and standard deviation. The profiling of antibiotics on each microorganism in the presence or absence of GTP or LTP was established.

The percentage of increase/decrease of combination treatment was calculated as Eq.1 where $A$ is zone of inhibition of combined treatment and $B$ represents the zone of inhibition of respective antibiotics [12]:

$$
\%=\frac{A-B}{B} \times 100
$$

A: ZOI of combined treatment; $B$ : ZOI of respective antibiotics.

Table 1 is the reference chart derived from Clinical Laboratory Standards Institute to determine the classification of the antibiotics per given microorganism [11]. Variation occurs among microorganisms, however, it provides a good consensus to establish a quick, efficient profiling schematic to further determine if any effects have occurred $(+/-$ in percentage). Afterwards, closer examination and research can be done to determine positive versus negative impacts in our research of combinative polyphenol antibiotic supplementation.

\section{RESULTS}

The effects of 12 antibiotics, alone or in combination, with one of the tea polyphenols (GTP or LTP) on 6 dif- 


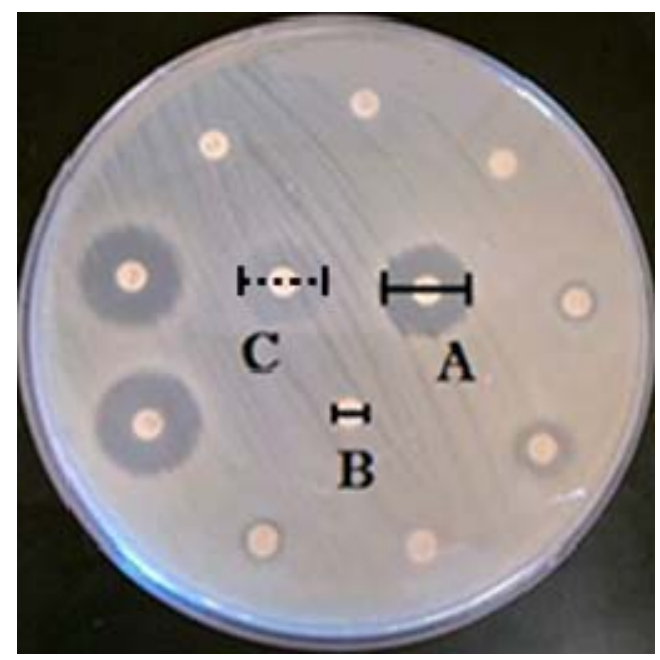

Figure 1. Disk diffusion susceptibility test used for the Kirby-Bauer testing with antibiotic disks infused with the compound of interest. A: Complete zone of inhibition (ZOI); B: No ZOI; C: Partial/weak ZOI.

Table 1. Kirby-Bauer disk diffusion categories of susceptible $(\mathrm{S})$, intermediate (I) and resistant (R).

\begin{tabular}{ccccc}
\hline $\begin{array}{c}\text { Antimicrobial } \\
\text { agents }\end{array}$ & $\begin{array}{c}\text { Disk } \\
\text { potency }\end{array}$ & $\begin{array}{c}\text { Resistant } \\
(\mathrm{mm})\end{array}$ & $\begin{array}{c}\text { Intermediate } \\
(\mathrm{mm})\end{array}$ & $\begin{array}{c}\text { Susceptible } \\
(\mathrm{mm})\end{array}$ \\
\hline $\begin{array}{c}\text { Ampicillin } \\
\text { (AM10) }\end{array}$ & $10 \mu \mathrm{g}$ & $<12$ & $12-13$ & $>13$ \\
$\begin{array}{c}\text { Bacitracin } \\
\text { (B10) }\end{array}$ & $10 \mu \mathrm{g}$ & $<9$ & $9-12$ & $>12$ \\
$\begin{array}{c}\text { Cephalothin } \\
\text { (CF30) }\end{array}$ & $30 \mu \mathrm{g}$ & $<15$ & $15-17$ & $>17$ \\
$\begin{array}{c}\text { Chloramphenicol } \\
\text { (C30) }\end{array}$ & $30 \mu \mathrm{g}$ & $<13$ & $13-17$ & $>17$ \\
$\begin{array}{c}\text { Doxycycline } \\
\text { (D30) }\end{array}$ & $30 \mu \mathrm{g}$ & $<15$ & $15-16$ & $>16$ \\
$\begin{array}{c}\text { Erythromycin } \\
\text { (E15) }\end{array}$ & $15 \mu \mathrm{g}$ & $<14$ & $14-17$ & $>17$ \\
$\begin{array}{c}\text { Gentamicin } \\
\text { (GM10) }\end{array}$ & $10 \mu \mathrm{g}$ & $<15$ & $14-17$ & $>17$ \\
$\begin{array}{c}\text { Penicillin } \\
\text { (P10) }\end{array}$ & $10 \mu \mathrm{g}$ & $<12$ & $12-21$ & $>21$ \\
$\begin{array}{c}\text { Polymyxin } \\
\text { (PB300) }\end{array}$ & $300 \mu \mathrm{g}$ & $<9$ & $9-11$ & $>11$ \\
$\begin{array}{c}\text { Rifampin } \\
\text { (RA5) }\end{array}$ & $5 \mu \mathrm{g}$ & $<12$ & $12-14$ & $>14$ \\
$\begin{array}{c}\text { Streptomycin } \\
\text { (S10) }\end{array}$ & $10 \mu \mathrm{g}$ & $<12$ & $12-14$ & $>14$ \\
$\begin{array}{c}\text { Tetracycline } \\
\text { (TE30) }\end{array}$ & $30 \mu \mathrm{g}$ & $<15$ & $15-18$ & $>18$ \\
\hline & & & &
\end{tabular}

ferent bacteria were evaluated. Profiling of these studies was generated from zone of inhibition, \% of increase/ decrease, and comparative analysis in determining inhibition efficiency versus the antibiotics alone. The results also indicate whether the synergism of GTP or LTP possesses the possibility to convert original antibiotic resis- tant to antibiotic sensitive.

\subsection{Gram-Positive Microorganisms}

Two gram-positive bacteria, S. epidermidis and B. megaterium, were used in profiling the combination effect of green tea polyphenol with different antibiotics. S. epidermidis is a normal flora on our skin, however, given certain conditions can become a potential pathogen. $B$. megaterium is a gram-positive bacterium and endospore producer. These spores produced by Bacillus are a main concern for hospitals in the medical community and the food industry due to its difficulty in eradication [13]. The results from this profiling study are shown in Figure 2(a) and the \% of increase or decrease with GTP or LTP on the effect of antibiotics are shown in Figure 2(b). One percent of GTP and LTP increased antibiotic efficacy ranging from $17 \%$ to $213 \%$ and $11 \%-183 \%$ respectively. Rifampin is noted to have the most significant increase in polyphenol/antibiotic inhibitive efficacy being $213 \%$ and $183 \%$ for RA5 for GTP and LTP respectively. Combination of GTP with AM10, B10, C30 and RA5 and combination of LTP with C30 and RA5 increased the efficacy of these antibiotics more than $100 \%$. The results indicate that both GTP and LTP have synergistic effect on all the antibiotics except PB300 for S. epidermidis. 1\% LTP is able to convert $S$. epidermidis resistant to $\mathrm{C} 30$ and RA5 into susceptible. 1\% GTP can work with AM10, B10, C30, P10 and RA5 to convert antibiotics that are not effective to having an impact against this microorganism.

In the study of B. megaterium, shown in Figures 3(a) and (b), GTP was found to increase antibiotic efficiency from $5 \%$ to $112 \%$ except on doxycycline (D30). LTP had a range from $3 \%$ to $55 \%$ with many negative impacts. The result revealed that GTP had an inhibition of $112 \%$ with B10. Some negative impacts of adding GTP or LTP on the antibiotics that were observed may be due to interaction of the tea polyphenols with antibiotics, which reduce the activities of antibiotics. The results suggest that GTP has a significant synergistic effect on antibiotics for Bacillus than LTP. Although GTP and LTP were able to increase the inhibition of some antibiotics, they were not able to convert the status from antibiotic resistant to sensitive for all the antibiotics used in this study.

\subsection{Acid-Fast Microorganism}

The results of profiling the effect of $1 \%$ of GTP and LTP with antibiotics on M. smegmatis are shown in Figures 4(a) and (b). The effects of GTP and LTP had ranges of $9 \%$ to $89 \%$ and $83 \%$ to $125 \%$ respectively. The highest percentage of inhibition was observed with $1 \%$ LTP in combination with P10 and AM10.

GTP was shown to have a synergistic effect on broader-antibiotics but only changed resistant acid-fast mi- 


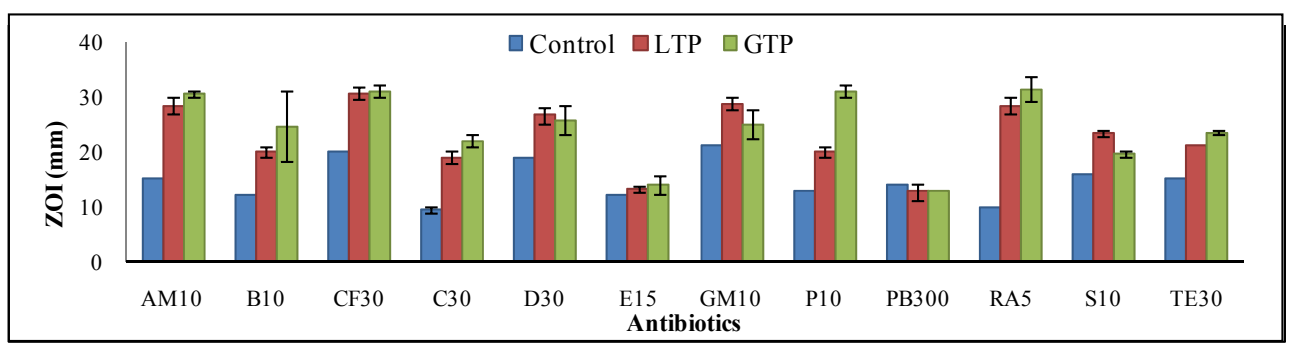

(a)

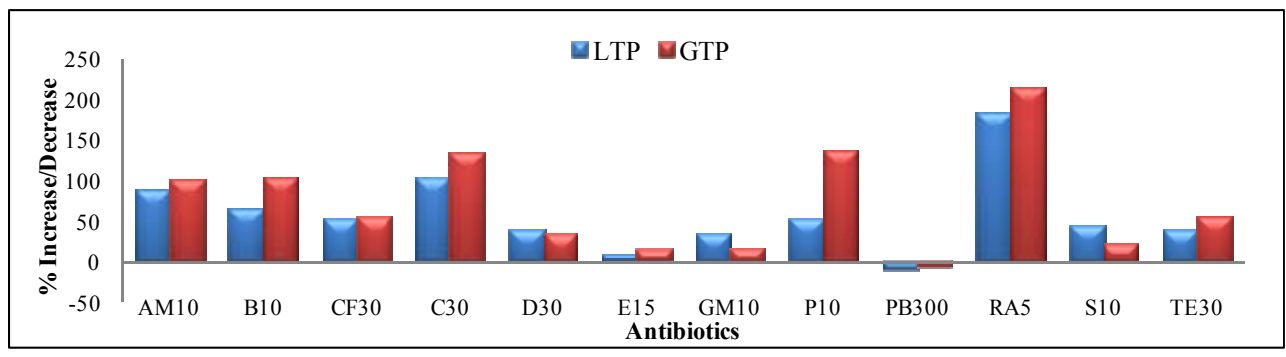

(b)

Figure 2. Profiling of Staphylococcus epidermidis of antibiotics alone and in combination with either $1 \%$ LTP or $1 \%$ GTP. (a) Zone of inhibition measured in $\mathrm{mm}$; (b) Percentage increase/decrease of antibiotics with LTP and GTP.

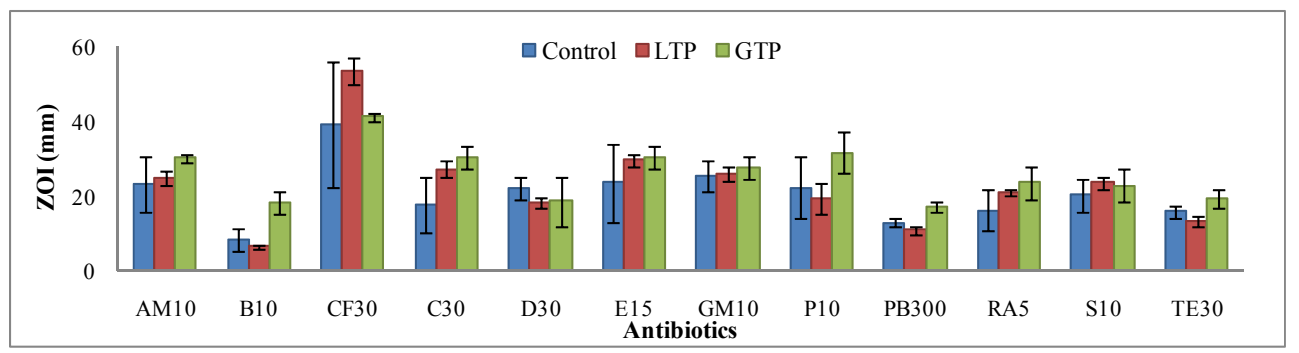

(a)

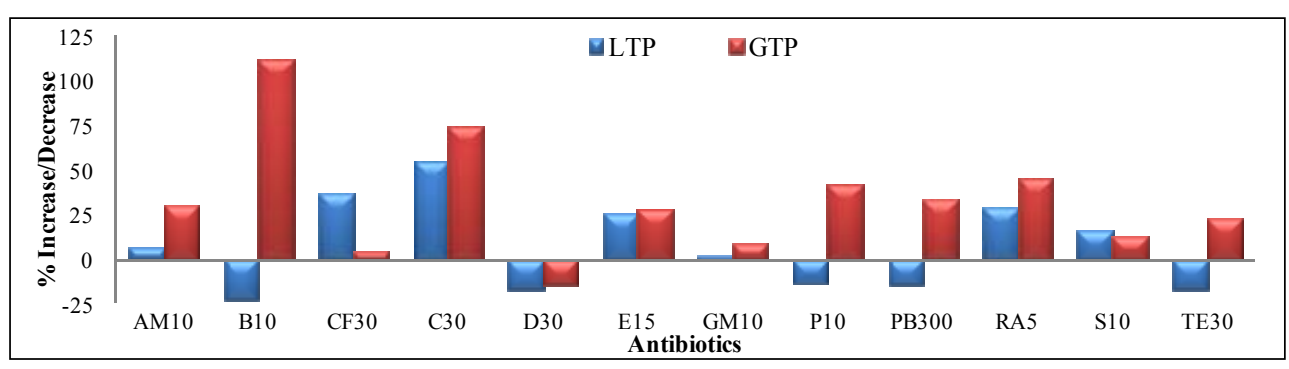

(b)

Figure 3. Profiling of Bacillus megaterium on antibiotics alone and in combination with either $1 \%$ LTP or $1 \%$ GTP. (a) Zone of inhibition measured in $\mathrm{mm}$; (b) Percentage increase/decrease of antibiotics with LTP and GTP.

croorganisms to susceptible when used in combination with E15. LTP showed a better infusion with targeted antibiotics AM10, B10, CF30, and P10 as well as converted the status of antibiotic resistant to susceptible.

\subsection{Gram-Negative Microorganism}

E. coli is part of the normal flora in our intestine; however, it can cause infection in invasive wounds, urinary tract infections, abdominal cavity ailments, diarrhea and many other diseases [9]. E. coli showed great affinity for the usage of LTP over GTP as seen in Figures 5(a) and (b). E. coli has been shown to have resistance to AM10, B10, C30, D30, E15, P10, PB300 and TE30. The effects of LTP remarkably reversed all these resistance, and enabled an increase in the zone of inhibition ranging from $28 \%$ to $161 \%$. It increased the inhibition of AM10, B10, 


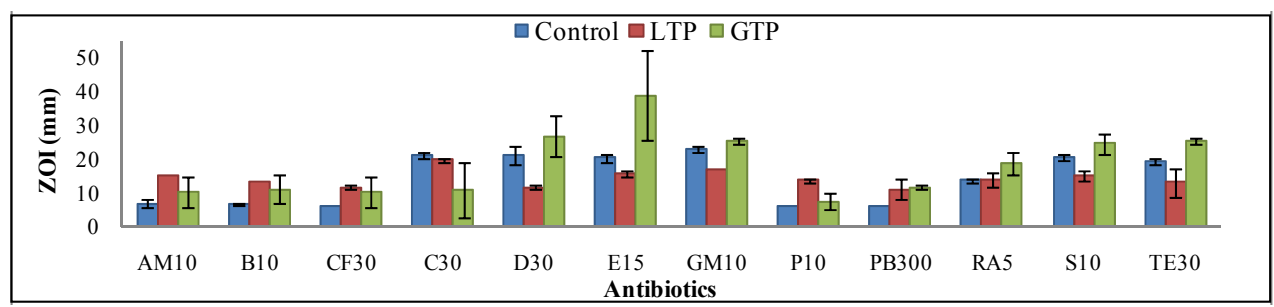

(a)

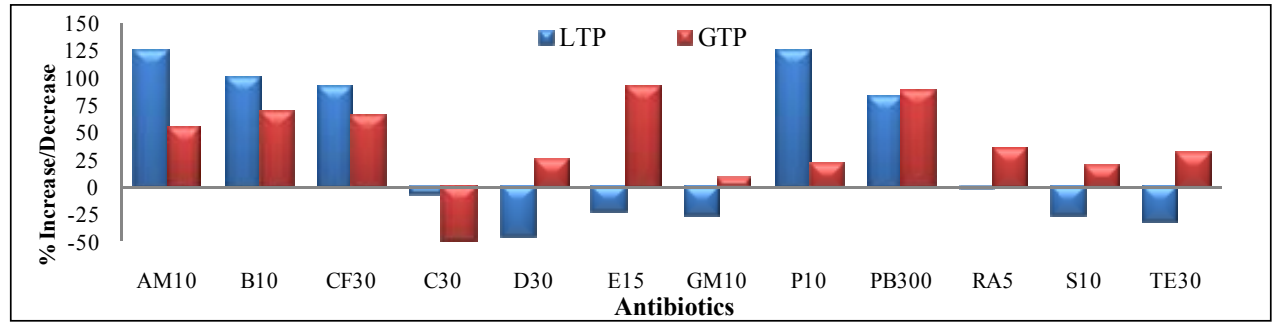

(b)

Figure 4. Profiling of Mycobacterium smegmatis of antibiotics alone and in combination with either $1 \%$ LTP or $1 \%$ GTP. (a) Zone of inhibition measured in mm; (b) Percentage increase/decrease of antibiotics with LTP and GTP.

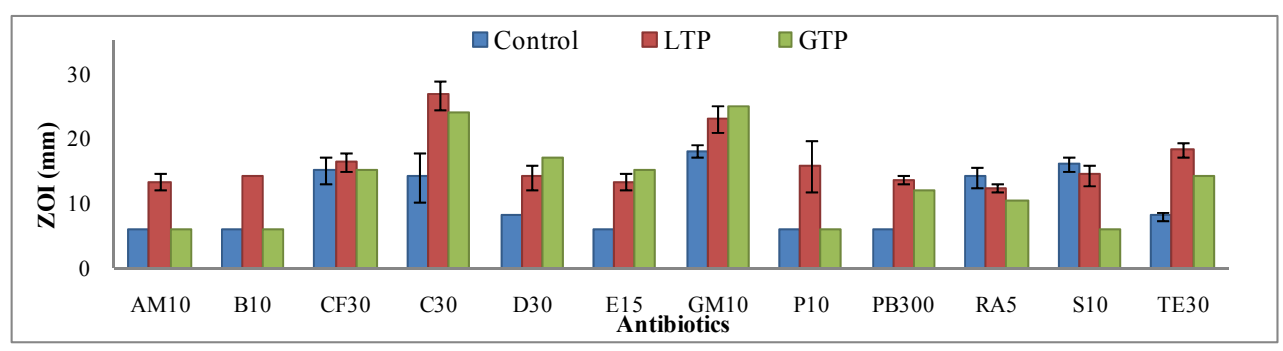

(a)

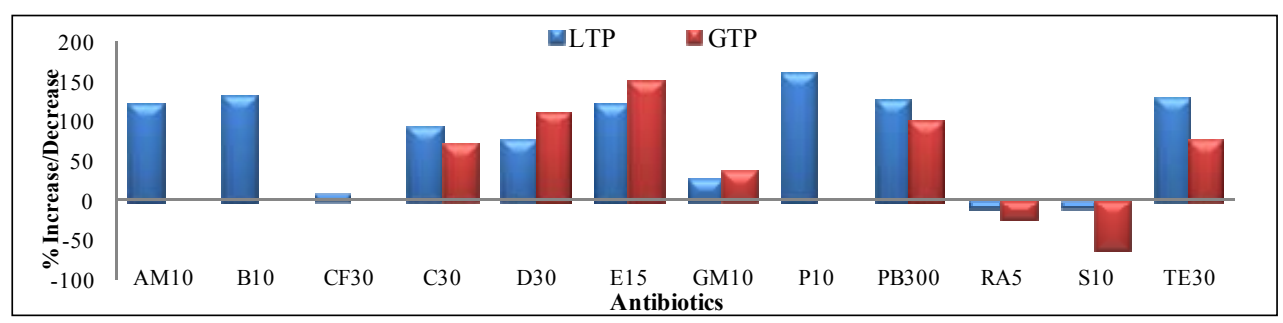

(b)

Figure 5. Profiling of Escherichia coli of antibiotics alone and in combination with either 1\% LTP or $1 \%$ GTP. (a) Zone of inhibition measured in $\mathrm{mm}$; (b) Percentage increase/decrease of antibiotics with LTP and GTP.

E15, P10, PB300 and TE30 more than 100\%. GTP did not have an impact on such a broad spectrum of antibiotics, as aforementioned antibiotics, but did have more than $100 \%$ on D10, E15, and PB300. Therefore, it was shown that 1\% LTP converted the effect of AM10, B10, C30 E15, P10, PB300, and TE30 on E. coli from resistant to susceptible, while GTP had synergistic effects with C30, D30, E15, and PB300.

$S$. marcescens, a red-pigmented gram-negative microorganism found in nosocomial infections and is an opportunistic human pathogen, is a very resistant microor- ganism [14]. This study indicated that S. marcescens is very resistant to most of the antibiotics. It was found that GTP did not produce favorable results; LTP had a tremendous impact with B10, CF30, and RA5 with 292\%, $119 \%$, and $225 \%$ increase inhibition respectively. In the presence of LTP, S. marcescens resistance was converted to susceptible on these antibiotics as shown in Figures 6(a) and (b).

E. aerogenes, a gram-negative microorganism, is known to cause many hospitals acquired bacterial infections [15]. The results indicated that GTP works with all twelve 


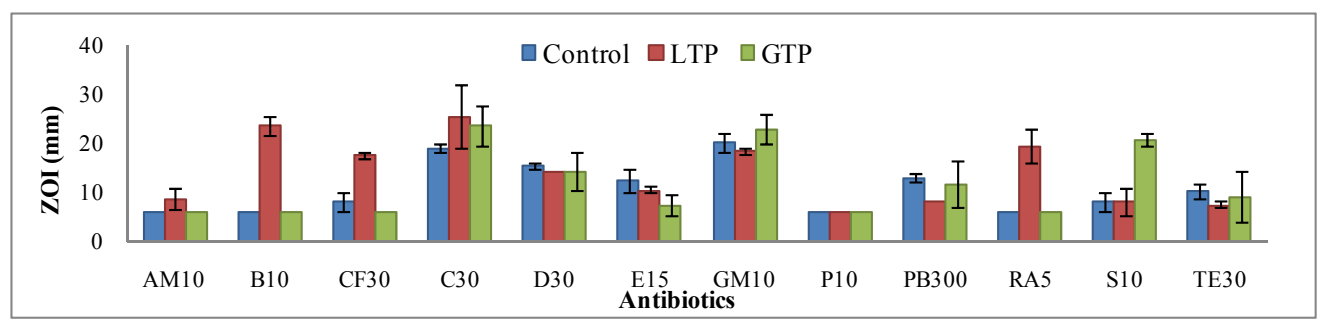

(a)

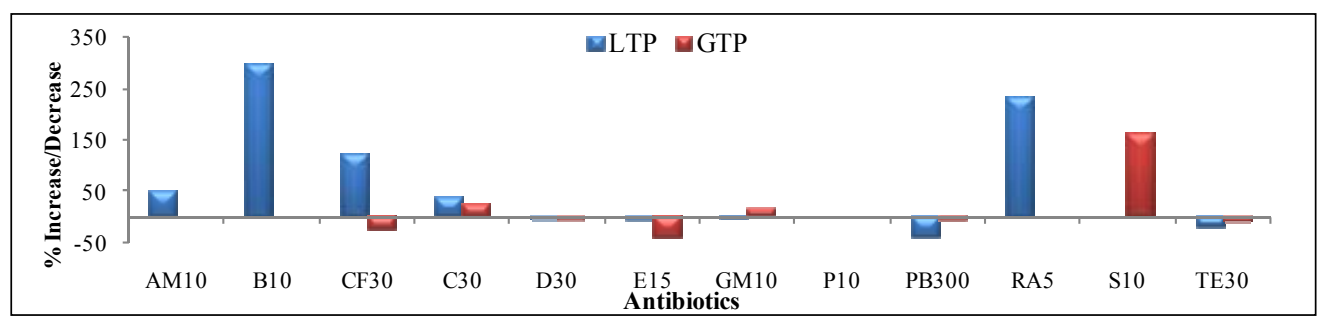

(b)

Figure 6. Profiling of Serratia marcescens of antibiotics alone and in combination with either 1\% LTP or $1 \%$ GTP. (a) Zone of inhibition measured in $\mathrm{mm}$; (b) Percentage increase/decrease of antibiotics with LTP and GTP.

antibiotics; with a $406 \%$ increase inhibition compare with the AM10 alone and 359\% increase inhibition for CF30 and over $100 \%$ of increase inhibition on B10, D30, E15, P10, PB300, RA5 and TE30. On the other hand, LTP had made negative impacts on many of the antibiotics. It was shown to only works on E15 and TE30 with the increase percentage being $119 \%$ and $233 \%$ respectively converting resistance to susceptibility (Figures 7(a) and (b)).

Table 2 showed that $1 \%$ GTP worked the best with $E$. aerogenes compared to other microorganisms. It was able to synergistically work with all nine antibiotics with percentages of inhibition above $100 \%$. The greatest percent of inhibition for E. aerogenes was $406 \%$. GTP also works very well with $S$. epidermidis where it had percentages greater than $100 \%$ for five antibiotics. GTP only had effects on two antibiotics for S. marcescens. The data indicates values of percentage against the microorganisms. The highest value is Ampicillin at 359\% against the gram-negative microorganism E. aerogenes. Any value higher than $100 \%$ is favorable. S. epidermidis had several antibiotics that showed to have a $100 \%$ or greater ZOI in usage with GTP.

Table 3 gave an overview summary of inhibition for each microorganism with 1\% LTP in combination with the twelve antibiotics. The data with a numerical value of $100 \%$ or greater were the most desired for further analysis and documentation. 1\% LTP appeared to inhibit E. coli, E. aerogenes, S. marcescens, and M. smegmatis with the most antibiotics. This suggested that $1 \%$ LTP in combination with antibiotics seemed to inhibit gramnegative microorganisms preferably; a very significant finding due to gram-negative bacteria's composition and poorer response to antibiotic treatment. E. coli had several antibiotics that showed to have a $100 \%$ or greater ZOI in usage with LTP.

In the presence of $1 \%$ GTP or $1 \%$ LTP, the polyphenols were able to convert some microorganisms from their antibiotic resistant origins to a nature of antibiotic susceptibility as shown in Table 4. The results indicated that the 1\% LTP worked on E. coli and converted seven antibiotics that were categorized as resistant to susceptible. 1\% GTP worked the best synergistically against $E$. aerogenes, making the once resistant E. aerogenes become susceptible to the eight antibiotics. It is also demonstrated that only $1 \%$ LTP were able to inhibit the growth of highly antibiotic resistant $S$. marcescens synergistically with three antibiotics.

\section{DISCUSSION}

The synergistic effects of GTP and LTP varied depending upon microorganism, strain, classification, and antibiotic used against certain strains. The GTP and LTP compounds were very effective against gram-negative bacteria $E$. coli.

One of the most significant findings for this experiment was that the usage of the LTP was found to have a significant impact and quite successful in producing a therapeutic in vitro effect against $S$. marcescens, a highly resistant bacterium. The effectiveness of GTP and LTP were found to work prominently against $E$. coli and $S$. epidermidis, which served as models for severe pathogenic microorganisms that raid clinics worldwide.

It is also notable to understand that it is favorable to use LTP for E. coli and GTP for S. epidermidis. This suggests that the promotion of LTP could be used for 
Table 2. Percentage increase/decrease for individual antibiotic with GTP. A: E. coli, B: S. marcescens, C: E. aerogenes, D: S. epidermidis, E: B. megaterium, F: M. smegmatis.

\begin{tabular}{ccccccc}
\hline \% & A & B & C & D & E & F \\
\hline AM10 & 0 & 0 & 406 & 103 & 30 & 55 \\
B10 & 0 & 0 & 162 & 106 & 112 & 69 \\
CF30 & 0 & -25 & 359 & 55 & 5 & 67 \\
C30 & 71 & 26 & 70 & 136 & 74 & -49 \\
D30 & 113 & -6 & 167 & 36 & -15 & 27 \\
E15 & 150 & -41 & 237 & 17 & 28 & 92 \\
GM10 & 39 & 15 & 73 & 19 & 9 & 9 \\
P10 & 0 & 0 & 252 & 138 & 42 & 22 \\
PB300 & 100 & -7 & 189 & -7 & 33 & 89 \\
RA5 & -25 & 0 & 137 & 213 & 45 & 37 \\
S10 & -63 & 162.5 & 92 & 23 & 13 & 20 \\
TE30 & 75 & -10 & 228 & 57 & 23 & 32 \\
\hline
\end{tabular}

Table 3. Percentage increase/decrease for individual antibiotic with LTP. A: E. coli, B: S. marcescens, C: E. aerogenes, D: S. epidermidis, E: B. megaterium, F: M. smegmatis.

\begin{tabular}{ccccccc}
\hline \% & A & B & C & D & E & F \\
\hline AM10 & 122 & 42 & 0 & 89 & 7 & 125 \\
B10 & 133 & 292 & -14 & 67 & -23 & 100 \\
CF30 & 9 & 119 & -33 & 53 & 36 & 92 \\
C30 & 90 & 34 & -22 & 104 & 55 & -7 \\
D30 & 75 & -9 & 0 & 40 & -17 & -45 \\
E15 & 122 & -15 & 119 & 11 & 25 & -23 \\
GM10 & 28 & -8 & 15 & 37 & 3 & -26 \\
P10 & 161 & 0 & -33 & 54 & -13 & 125 \\
PB300 & 128 & -38 & 28 & -10 & -15 & 83 \\
RA5 & -12 & 225 & 30 & 183 & 29 & -1 \\
S10 & -10 & 0 & 47 & 46 & 16 & -26 \\
TE30 & 129 & -27 & 233 & 40 & -17 & -32 \\
\hline
\end{tabular}

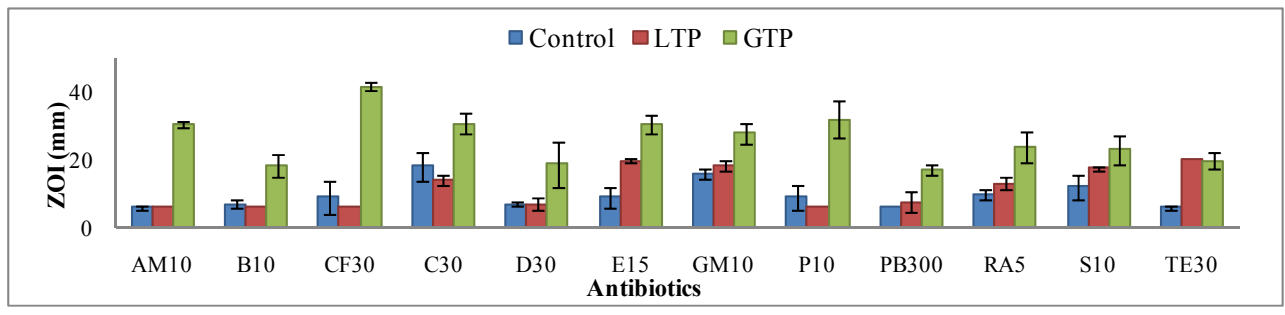

(a)

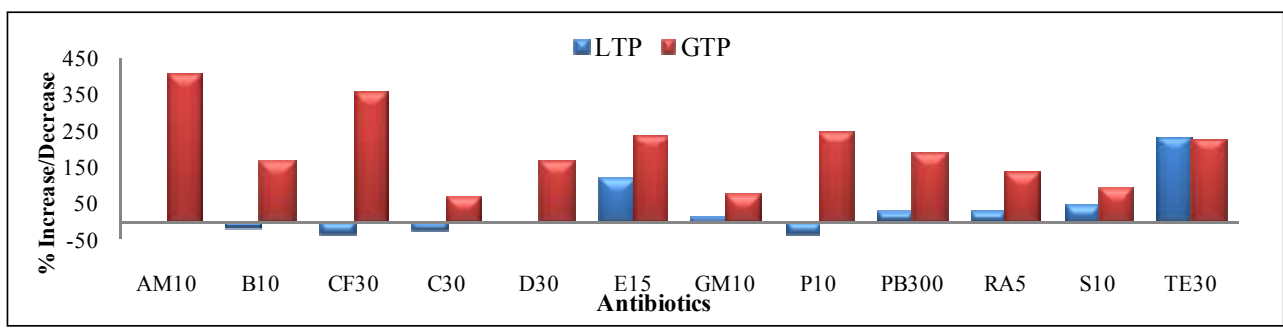

(b)

Figure 7. Profiling of Enterobacter aerogenes of antibiotics alone and in combination with either 1\% LTP or $1 \%$ GTP. (a) Zone of inhibition measured in $\mathrm{mm}$; (b) Percentage (\%) of increase/decrease of antibiotics with LTP and GTP.

Table 4. Comparison chart of resistant to susceptibility against antibiotics with GTP and LTP.

\begin{tabular}{|c|c|c|}
\hline Microorganisms & $1 \%$ GTP & $1 \%$ LTP \\
\hline E. coli & $\begin{array}{c}\mathrm{C} 30(71 \% \mathrm{R} \rightarrow \mathrm{S}), \mathrm{D} 30(113 \% \mathrm{R} \rightarrow \mathrm{S}), \mathrm{E} 15(150 \% \mathrm{R} \rightarrow \mathrm{S}), \\
\text { PB300 }(100 \% \mathrm{R} \rightarrow \mathrm{S})\end{array}$ & $\begin{array}{c}\text { AM10 }(122 \% R \rightarrow S), B 10(133 \% R \rightarrow S), C 30(90 \% R \rightarrow S), \\
\text { E15 }(122 \% R \rightarrow S), \text { P10 }(161 \% R \rightarrow S), \\
\text { PB300 }(128 \% R \rightarrow S), \text { TE30 }(129 \% R \rightarrow S)\end{array}$ \\
\hline S. marcescens & $\mathrm{S} 10(\mathrm{R} \rightarrow \mathrm{S})$ & $\begin{array}{c}\mathrm{B} 10(292 \% \mathrm{R} \rightarrow \mathrm{S}), \mathrm{CF} 30(119 \% \mathrm{R} \rightarrow \mathrm{S}) \\
\mathrm{RA} 5(225 \% \mathrm{R} \rightarrow \mathrm{S})\end{array}$ \\
\hline E. aerogenes & $\begin{array}{c}\text { AM10 }(406 \% R \rightarrow S), \text { B10 }(162 \% R \rightarrow S), \text { CF30 }(359 \% R \rightarrow S), \\
\text { D30 }(167 \% R \rightarrow S), \text { E15 }(237 \% R \rightarrow S) \text {, P10 }(252 \% R \rightarrow S), \\
\text { PB300 }(189 \% R \rightarrow S), \text { RA5 }(137 \% R \rightarrow S), \text { TE30 }(228 \% R \rightarrow S)\end{array}$ & E15 $(119 \% R \rightarrow S)$, TE30 $(233 \% R \rightarrow S)$ \\
\hline S. epidermidis & $\begin{array}{c}\text { AM10 }(103 \% R \rightarrow S), B 10(106 \% R \rightarrow S), C 30(136 \% R \rightarrow S) \\
\text { P10 }(138 \% R \rightarrow S), R A 5(213 \% R \rightarrow S)\end{array}$ & C30 $(104 \% R \rightarrow S)$, RA5 $(183 \% R \rightarrow S)$ \\
\hline B. megaterium & Impact not significant & Impact not significant \\
\hline M. smegmatis & E15 $(92 \% R \rightarrow S)$ & $\begin{array}{c}\text { AM10 }(125 \% R \rightarrow S), B 10(100 \% R \rightarrow S) \\
\text { P10 }(125 \% R \rightarrow S)\end{array}$ \\
\hline
\end{tabular}


gram-negative bacteria, such as seen in S. marcescens, and GTP for gram-positive bacteria. The exception to this statement is found with E. aerogenes which seemed to be the most susceptible to GTP. In addition, 1\% GTP and $1 \%$ LTP provide different synergistic effect on different antibiotics in different bacteria. This suggests that the difference in the molecular structure of GTP and LTP could impact their mode of action in their biochemical mechanisms.

\section{CONCLUDING REMARKS}

In summary, the percentage inhibition of $1 \%$ GTP and $1 \%$ LTP with antibiotics against microorganisms are shown in Tables 2 and 3. Table 2 showed that 1\% GTP worked the best against $E$. aerogenes compared to the other microorganisms. $1 \%$ GTP was able to provide synergistic inhibition percentages above $100 \%$ with all 9 antibiotics. The highest synergy was found with Ampicillin and GTP at $406 \%$ against E. aerogenes. GTP did not show any major synergistic effects for $S$. marcescens except for S10. Table 3 gave an overview of inhibition for six microorganisms with $1 \%$ LTP in combination with the twelve antibiotics. Some of the combination treatments indicated a greater than $100 \%$ of inhibition. 1\% LTP appeared to inhibit E. coli, S. marcescens and M. smegmatis with the most antibiotics. Any value higher than $100 \%$ is noted to be favorable. Several antibiotics showed a $100 \%$ or greater ZOI when combined with LTP against E. coli. Both GTP and LTP increased inhibition with most antibiotics on S. epidermidis, but LTP only worked on C30 and RA5 and GTP worked on AM10, B10, C30, P10 and RA5.

Table 4 summarized that 1\% LTP or 1\% GTP in combination with different antibiotics resulted in conversion of antibiotic resistant to susceptible. 1\% GTP worked the best synergistically against $E$. aerogenes, making the once resistant $E$. aerogenes now susceptible to 8 antibiotics. $1 \%$ LTP worked the best on E. coli and converted 7 antibiotics resistant to susceptible. It is also demonstrated that only $1 \%$ LTP was able to inhibit the growth of $S$. marcescens synergistically with three antibiotics.

This study has established a profile by using $1 \%$ GTP and $1 \%$ LTP versus controls which have allowed us to evaluate the efficacy of the synergistic combination with these antibiotics. They were able to change some of the antibiotics from resistant to susceptible.

The mechanism of EGCG, the major green tea watersoluble polyphenol, is not yet fully understood. However, there is a definite correlation for EGCG and its affinity to the cell wall composition of bacteria [16]. This suggests that GTP has an effect on bacterial cell wall; meanwhile, LTP provides a very different profiling suggesting perhaps a different mechanism of lipid soluble tea polyphenol on bacteria.

\section{FUTURE STUDIES}

Pure hydrophilic green tea polyphenol (GTP) such as EGCG and pure lipophilic tea compound EGCG-stearate should be used to further evaluate the efficacy of the synergism of these polyphenols on different antibiotics. The minimum inhibitory concentrations (MIC) with different combinations of tea polyphenols and antibiotics should be determined to obtain the optimal condition for potential application of therapeutic treatment of infection. Furthermore, the synergy mechanisms of GTP, LTP and different antibiotics should also be studied. These findings could prove useful for further in vitro studies that potentiate to in vivo applications.

\section{ACKNOWLEDGEMENTS}

We deeply appreciated Dr. Stephen Hsu at Life Sciences Business Development Center, Georgia Regents University, Augusta, GA for providing GTP and LTP. This work was supported by Montclair State University Faculty Scholarship Program (FSP) to LHL; Novartis Scholarship for graduate research to $\mathrm{UH}$; Science Honors Innovation Program (SHIP) for undergraduate research to HT; William \& Doreen Wong Foundation Grant and Seton Hall University Biological Sciences Research Fund to TC.

\section{REFERENCES}

[1] Si, W., Gong, J., Tsao, R., Kalab, M., Yang, R. and Yin, Y. (2006) Bioassay-guided purification and identification of antimicrobial components in Chinese green tea extract. Journal of Chromatography A, 1125, 204-210. http://dx.doi.org/10.1016/i.chroma.2006.05.061

[2] Mak, J.C. (2012) Potential role of green tea catechins in various disease therapies: Progress and promise. Clinical and Experimental Pharmacology and Physiology, 39, 265-273. http://dx.doi.org/10.1111/j.1440-1681.2012.05673.x

[3] Sanz Barbero, B. and Blasco Hernandez, T. (2007) Resistant Mycobacterium tuberculosis strains from immigrants in the community of Madrid: Current assessment. Archivos de Bronconeumología, 43, 324-333. http://dx.doi.org/10.1016/S1579-2129(07)60078-0

[4] An, B.J., Kwak, J.H., Son, J.H., Park, J.M., Lee, J.Y., Jo, C. and Byun, M.W. (2004) Biological and anti-microbial activity of irradiated green tea polyphenols. Food Chemistry, 88, 549-555.

http://dx.doi.org/10.1016/j.foodchem.2004.01.070

[5] Pernot, L., Frenois, F., Rybkine, T., L'Hermite, G., Petrella, S., Delettre, J., Jarlier, V., Collatz, E. and Sougakoff, W. (2001) Crystal structures of the class D betalactamase OXA-13 in the native form and in complex with meropenem. Journal of Molecular Biology, 310, 859-874. http://dx.doi.org/10.1006/jmbi.2001.4805

[6] Mori, S., Miyake, S., Kobe, T., Nakaya, T., Fuller, S.D., Kato, N. and Kaihatsu, K. (2008) Enhanced anti-influenza A virus activity of (-)-epigallocatechin-3-O-gallate 
fatty acid monoester derivatives: Effect of alkyl chain length. Bioorganic \& Medicinal Chemistry Letters, 18, 4249-4252. http://dx.doi.org/10.1016/j.bmcl.2008.02.020

[7] Chen, P., Dickinson, D. and Hsu, S. (2009) Lipid-soluble green tea polyphenols:stabilized for effective formulation. In: McKinley, H. and Jamieson, M., Eds., Handbook of Green Tea and Health Research, Food and Beverage Consumption and Health, 45-61.

[8] Chen, P., Tan, Y., Sun, D. and Zheng, X.M. (2003) A novel long-chain acyl-derivative of epigallocatechin-3O-gallate prepared and purified from green tea polyphenols. Journal of Zhejiang University Science, 4, 714-718. http://dx.doi.org/10.1631/jzus.2003.0714

[9] Zscheck, K.K. and Murray, B.E. (1993) Genes involved in the regulation of beta-lactamase production in enterococci and staphylococci. Antimicrobial Agents and Chemotherapy, 37, 1966-1970.

http://dx.doi.org/10.1128/AAC.37.9.1966

[10] Bauer, A.W., Kirby, W.M., Sherris, J.C. and Turck, M. (1966) Antibiotic susceptibility testing by a standardized single disk method. American Journal of Clinical Pathology, 45, 493-496.

[11] Clinical Laboratory Standards Institute (2006) Performance standards for antimicrobial disk susceptibility tests. Approved Standard, 9th Edition, 2006.
[12] Lee, L.H. and Chu, T.C. (2012) Microbiology laboratory manual. Hayden-McNeil Publishing.

[13] Ball, A.P., McGhie, D. and Geddes, A.M. (1977) Serratia marcescens in a general hospital. QJM: An International Journal of Medicine, 46, 63-71.

[14] Sainsbury, S., Bird, L., Rao, V., Shepherd, S.M., Stuart, D.I., Hunter, W.N., Owens, R.J. and Ren, J. (2011) Crystal structures of penicillin-binding protein 3 from Pseudomonas aeruginosa: Comparison of native and antibiotic-bound forms. Journal of Molecular Biology, 405, 173-184. http://dx.doi.org/10.1016/j.jmb.2010.10.024

[15] Nakayama, M., Shigemune, N., Tsugukuni, T., Jun, H., Matsushita, T., Mekada, Y., Kurahachi, M. and Miyamoto, T. (2012) Mechanism of the combined anti-bacterial effect of green tea extract and $\mathrm{NaCl}$ against Staphylococcus aureus and Escherichia coli O157:H7. Food Control, 25, 225-232. http://dx.doi.org/10.1016/j.foodcont.2011.10.021

[16] Zhao, W.H., Hu, Z.Q., Okubo, S., Hara, Y. and Shimamura, T. (2001) Mechanism of synergy between epigallocatechin gallate and beta-lactams against methicillinresistant Staphylococcus aureus. Antimicrobial Agents and Chemotherapy, 45, 1737-1742. http://dx.doi.org/10.1128/AAC.45.6.1737-1742.2001 